\title{
O papel do exercício físico no tratamento da enxaqueca: qual a evidência?
}

Maria João Coelho, ${ }^{1}$ Daniela Pereira, ${ }^{2}$ Filipa Paraíso, ${ }^{3}$ Tiago Costa ${ }^{4}$

\begin{abstract}
RESUMO
Objetivo: Determinar a evidência do efeito do exercício aeróbico no controlo das crises de enxaqueca.

Fontes de dados: PubMed, National Institute of Health and Care Excellence (NICE), The Cochrane Library, British Medical Journal (BMJ), Canadian Medical Association Infobase e Centre for Reviews and Dissemination.

Métodos: Foram pesquisados artigos de revisão sistemática (RS), meta-análises (MA) e normas de orientação clínica (NOC), em português, inglês e espanhol, sem limite de data de publicação, utilizando os termos MeSH migraine disorders e exercise. Foram também pesquisados estudos originais (EO) publicados nos últimos cinco anos. Incluíram-se os artigos que avaliavam o efeito do exercício aeróbico na frequência, duração e intensidade das crises de enxaqueca. A atribuição do nível de evidência (NE) e força de recomendação (FR) foi realizada com base na escala Strength of Recommendation Taxonomy (SORT), da American Academy of Family Physicians.

Resultados: Da pesquisa efetuada obtiveram-se 69 artigos, tendo sido selecionados seis que cumpriam os critérios de inclusão: três RS com MA, uma RS e dois EO. Todos os artigos apresentaram NE 2. A maioria dos estudos incluídos favorece a prática de exercício físico como medida profilática das crises de enxaqueca; contudo, apresentam amostras pequenas, curtos períodos de intervenção e de follow-up e intervenções heterogéneas.

Conclusão: Apesar de se considerar necessária a realização de mais ensaios clínicos para definir concretamente os efeitos a curto e longo prazo do exercício aeróbico na enxaqueca conclui-se que é possível recomendar a sua inclusão na abordagem profilática destes doentes (FR B).
\end{abstract}

Palavras-chave: Enxaqueca; Exercício físico.

\section{INTRODUÇÃO}

1 s cefaleias primárias constituem uma das patologias mais prevalentes em todo o mundo. ${ }^{1-2}$ A enxaqueca corresponde a $69 \%$ das cefaleias primárias, tendo uma prevalência estimada de cerca de $15 \%$ e é uma das principais causas de incapacidade mundial. ${ }^{1,3-4}$ Estudos revelam que a perda de qualidade de vida dos doentes com enxaqueca é tão grave quanto a verificada na depressão e ainda mais severa do que a que ocorre em outras doenças crónicas, como a diabetes e a lombalgia. ${ }^{5-6}$ A enxaqueca não é apenas um problema de saúde com enorme impacto a nível pessoal, mas também a nível socioeconómico,

1. Médica Interna de Medicina Geral e Familiar. USF Freamunde. Freamunde, Portugal. 2. Médica Interna de Medicina Geral e Familiar. USF Salvador Lordelo. Lordelo, Portugal. 3. Médica Interna de Medicina Geral e Familiar. USF Santa Luzia. Freamunde, Portugal. 4. Médico Interno de Medicina Geral e Familiar. USF Hygeia. Vila Cova de Lixa, Portugal. sendo responsável por um elevado absentismo laboral nos países ocidentais, com custos anuais médios na Europa estimados em 27 biliões de euros. ${ }^{2,8-9}$

Do ponto de vista terapêutico, a abordagem da enxaqueca divide-se em tratamento agudo e, nos casos em que o mesmo se justifique, tratamento profilático. Para o tratamento agudo utilizam-se geralmente analgésicos como os anti-inflamatórios não esteroides e triptanos. ${ }^{9-10}$ O tratamento profilático, por sua vez, está recomendado se existirem mais de três episódios mensais de enxaqueca moderada a grave, se os sintomas persistirem mais de oito dias por mês, apesar de tratamento farmacológico otimizado, e se o doente é refratário ou tem contraindicações para realização de tratamento agudo. ${ }^{11}$ A necessidade desse tipo de tratamento poderá estar associada a vários riscos, como o dos efeitos adversos e o da sobredosagem. ${ }^{12}$ Neste contexto existem 


TABELA 1. População/Intervenção/Comparativo/Outcome (PICO) da revisão
\begin{tabular}{l|l} 
PICO \\
\hline População & Indivíduos com diagnóstico de enxaqueca, com idade igual ou superior a 18 anos, independentemente do género \\
\hline Intervenção & Prática de exercício físico aeróbico \\
\hline Comparação & Ausência de intervenção \\
\hline Outcome & Redução da frequência, duração e intensidade das crises de enxaqueca \\
\hline
\end{tabular}

estratégias não farmacológicas complementares ou alternativas de tratamento que têm como objetivo melhorar o controlo da enxaqueca e, consequentemente, a capacidade funcional destes indivíduos, minimizando o risco de efeitos adversos do tratamento profilático farmacológico já referidos. ${ }^{13}$ Assim, a prática de exercício aeróbico de intensidade moderada é sugerida como possível tratamento profilático, pois parece desempenhar um papel importante na modulação da dor, com possível efeito analgésico a curto e longo prazo, quer a nível central quer periférico. ${ }^{11,14-15}$

Nos últimos anos têm sido publicados vários estudos sobre o efeito do exercício físico na dor crónica. ${ }^{16}$ Do mesmo modo, a última versão da Classificação Internacional de Cefaleias (ICHD-III) salienta a necessidade de rever os efeitos do exercício aeróbico na profilaxia da enxaqueca. ${ }^{11,17}$

Esta revisão tem como objetivo determinar a evidência do efeito do exercício aeróbico no controlo das crises de enxaqueca, nomeadamente na sua frequência, duração e intensidade da dor durante as crises.

\section{MÉTODOS}

Em 2 de fevereiro de 2020 foi realizada uma pesquisa de normas de orientação clínica (NOC), artigos de revisão sistemática (RS), meta-análises (MA) e estudos originais (EO) em websites de medicina baseada na evidência, nomeadamente PubMed, National Institute of Health and Care Excellence (NICE), The Cochrane Library, British Medical Journal(BMJ), Canadian Medical Association Infobase e Centre for Reviews and Dissemination. Os termos MeSH utilizados foram migraine disorders e exercise. A pesquisa não foi limitada pela data de publicação, à exceção dos EO, dos quais apenas foram avaliados os artigos publicados a partir do ano de 2015, inclusive.
Foram incluídos os artigos de língua portuguesa, inglesa e espanhola realizados na população adulta ( $\geq 18$ anos) com diagnóstico de enxaqueca (população). Os estudos incluídos deveriam ter um grupo submetido à prática de exercício físico aeróbico (intervenção), comparativamente a um grupo placebo/ausência de exercício aeróbico (comparação). O resultado medido (outcome) foi o efeito do exercício físico aeróbico na frequência, duração e intensidade das crises de enxaqueca (Tabela 1). Foram excluídos os artigos duplicados, EO incluídos nas RS e MA utilizadas, artigos que não respondiam ao modelo PICO bem como artigos com tipologias não passíveis de inclusão no presente trabalho (Figuras 1 e 2). A seleção dos trabalhos por título e resumo foi efetuada por dois autores de modo independente. Os artigos selecionados para leitura integral foram lidos por pelo menos dois dos autores para decidir a sua inclusão em caso de dúvida. A avaliação final da qualidade e nível de evidência dos artigos incluídos foi discutida e decidida por consenso entre todos os autores. A atribuição do nível de evidência (NE) e força de recomendação (FR) foi realizada com base na escala Strength of Recommendation Taxonomy (SORT), da American Academy of Family Physicians. ${ }^{18}$

\section{RESULTADOS}

Através da pesquisa realizada obtiveram-se 69 artigos: 16 RS e 53 EO. Após aplicação da metodologia acima descrita resultaram seis artigos, nomeadamente três RS com MA (Tabela 2), uma RS (Tabela 3) e dois EO (Tabela 4$)$.

\section{Revisões sistemáticas com meta-análises}

La Touche e colaboradores ${ }^{19}$ concluíram que o exercício físico aeróbico pode diminuir a intensidade e a frequência das crises de enxaqueca, ainda que com baixa 


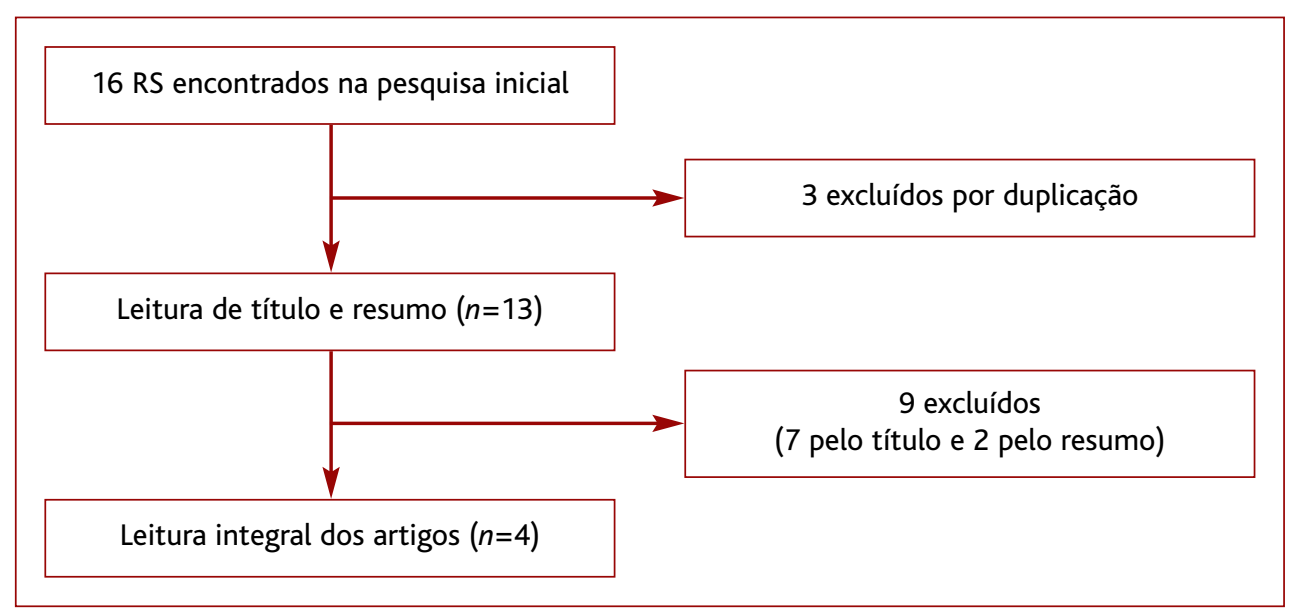

Figura 1. Metodologia de seleção de artigos - revisões sistemáticas.

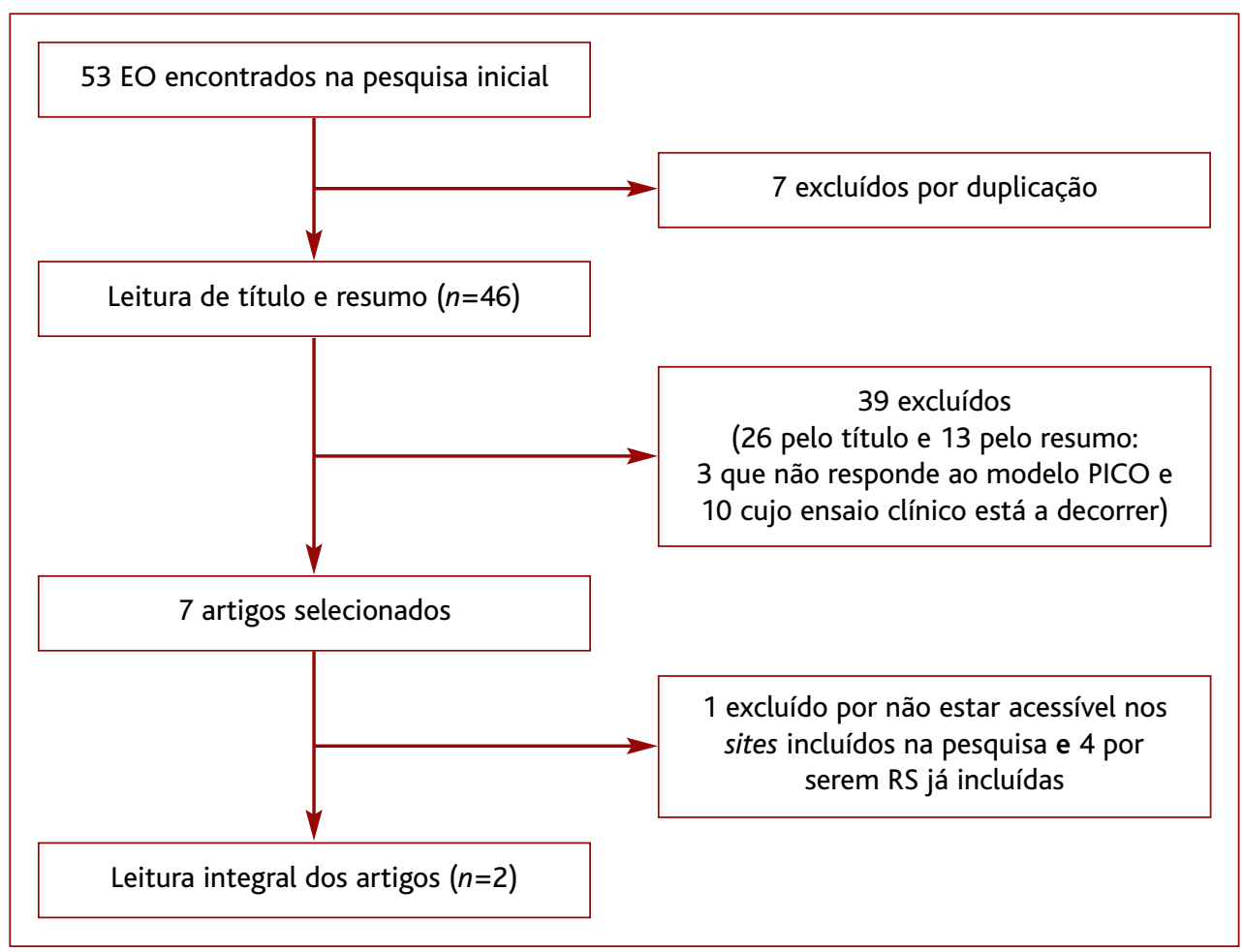

Figura 2. Metodologia de seleção de artigos - estudos originais.

qualidade de evidência, uma vez que os estudos incluídos apresentaram heterogeneidade nas intervenções e nos tempos de follow-up e ainda, em dois deles, ausência de dupla ocultação [Darabaneanu et al., 2011; Lockett et al., 1992].

Lemmens e colaboradores ${ }^{11}$ demonstraram que o exercício aeróbico parece diminuir o número de dias das crises bem como a sua intensidade e duração. Estes resultados têm, no entanto, limitações, uma vez que os estudos incluídos eram heterogéneos (por compararem diferentes tipos de exercício), apresentavam follow-up inadequado (com elevadas taxas de abandono) 
TABELA 2. Revisões sistemáticas + meta-análises

Revisão Sistemática + Meta-análise

\begin{tabular}{|c|c|c|c|c|c|c|}
\hline $\begin{array}{l}\text { Referência } \\
\text { bibliográfica }\end{array}$ & $\begin{array}{c}\text { Critérios } \\
\text { inclusão/exclusão }\end{array}$ & Estudos incluídos & $\begin{array}{l}\text { Intervenção (I) e } \\
\text { Comparação (C) }\end{array}$ & Resultados & Conclusão & NE \\
\hline $\begin{array}{l}\text { La Touche et } \\
\text { al. }(2020)^{19}\end{array}$ & $\begin{array}{l}\text { Inclusão: Adultos } \\
\text { com o diagnóstico } \\
\text { de enxaqueca com } \\
\text { ou sem aura, } \\
\text { segundo os } \\
\text { critérios da } \\
\text { Classificação } \\
\text { Internacional de } \\
\text { Cefaleias (ICHD). } \\
\text { Exclusão: não } \\
\text { descritos }\end{array}$ & $\begin{array}{l}7 \text { estudos } \\
\text { aleatorizados } \\
\text { controlados (EAC) } \\
\text { e } 3 \text { estudos não } \\
\text { aleatorizados } \\
\text { controlados } \\
\text { (ENAC): } \\
5 \text { respondem ao } \\
\text { PICO } \\
\text { - Lockett et al., } \\
\text { 1992 ( } n=20) \\
\text { - Dittrich et al., } \\
2008 \text { ( } n=30) \text { - } \\
\text { também incluído } \\
\text { na RS Gil-Martínez } \\
\text { et al., } 2013 \\
\text { - Kroll et al., 2018 } \\
\text { ( } n=52 \text { ) } \\
\text { - Darabaneanu et } \\
\text { al., 2011 ( } n=16 \text { ) - } \\
\text { também incluído } \\
\text { na RS+MA } \\
\text { Lemmens et al., } \\
2019 \text { e de Luedtke } \\
\text { et al., } 2016 \\
\text { - Hanssen et al., } \\
2017 \text { ( } n=15 \text { ) - } \\
\text { também incluído } \\
\text { na RS+MA } \\
\text { Lemmens et al., } \\
2019\end{array}$ & 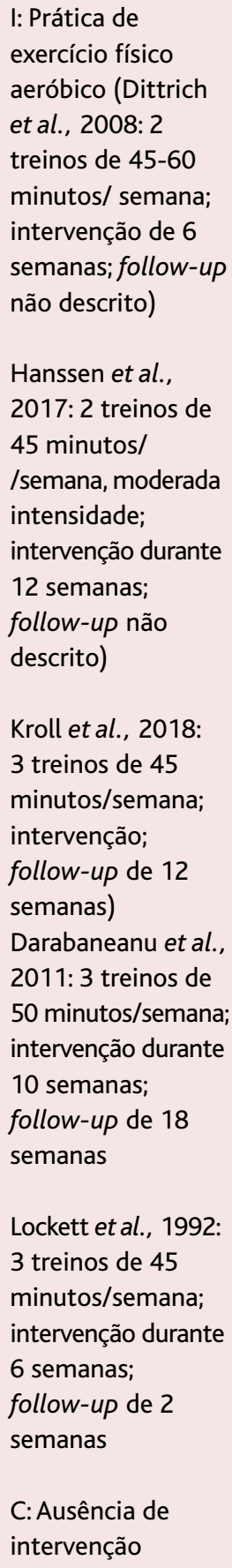 & $\begin{array}{l}\text { Dittrich et al., 2008: } \\
\text { - redução de } \\
\text { intensidade da dor } \\
\text { (escala 0-5) com } \\
p=0,024 \text { - valor } \\
\text { com significância } \\
\text { estatística } \\
\text { Hanssen et al., } \\
\text { 2017: - redução da } \\
\text { frequência } \\
\text { Kroll et al., 2018: } \\
\text { - redução da } \\
\text { frequência de 9,2 } \\
\text { dias para } 7,2 \text { dias } \\
\text { ( } p=0,025 \text { ) - valor } \\
\text { sem significância } \\
\text { estatística } \\
\text { Darabaneanu et al., } \\
2011:- \text { redução de } \\
\text { frequência e } \\
\text { intensidade } \\
\text { Lockett et al., 1992: } \\
\text { - redução de } \\
\text { intensidade da dor } \\
\text { - valor sem } \\
\text { significância } \\
\text { estatística }\end{array}$ & $\begin{array}{l}\text { Existe evidência de } \\
\text { baixa qualidade de } \\
\text { que o exercício } \\
\text { aeróbico pode } \\
\text { diminuir a } \\
\text { intensidade e a } \\
\text { frequência das } \\
\text { crises de } \\
\text { enxaqueca }\end{array}$ & 2 \\
\hline
\end{tabular}


TABELA 2. Revisões sistemáticas + meta-análises (continuação)

\begin{tabular}{|c|c|c|c|c|c|c|}
\hline \multicolumn{7}{|c|}{ Revisão Sistemática + Meta-análise } \\
\hline $\begin{array}{l}\text { Referência } \\
\text { bibliográfica }\end{array}$ & $\begin{array}{c}\text { Critérios } \\
\text { inclusão/exclusão }\end{array}$ & Estudos incluídos & $\begin{array}{l}\text { Intervenção (I) e } \\
\text { Comparação (C) }\end{array}$ & Resultados & Conclusão & NE \\
\hline $\begin{array}{l}\text { Lemmens et } \\
\text { al. (2019) }{ }^{11}\end{array}$ & $\begin{array}{l}\text { Inclusão: adultos } \\
\text { com diagnóstico } \\
\text { de enxaqueca com } \\
\text { ou sem aura } \\
\text { classificados } \\
\text { segundo a } \\
\text { Classificação } \\
\text { Internacional de } \\
\text { Cefaleias - II } \\
\text { (ICHD - II) } \\
\text { Exclusão: outros } \\
\text { tipos de cefaleia; } \\
\text { outros tipos de } \\
\text { intervenção }\end{array}$ & $\begin{array}{l}5 \text { EAC e } 1 \text { ENAC: } 2 \\
\text { respondem ao PICO } \\
\text { - Darabaneanu et } \\
\text { al., } 2011(n=16) \\
\text { e } \\
\text { - Hanssen et al., } \\
2017(n=15)\end{array}$ & $\begin{array}{l}\text { I: Prática de } \\
\text { exercício físico } \\
\text { aeróbico durante, } \\
\text { pelo menos, } 6 \\
\text { semanas } \\
\text { (Darabaneanu et } \\
\text { al., 2011: } 3 \text { treinos } \\
\text { de } 50 \text { minutos/ } \\
\text { /semana; } \\
\text { intervenção } \\
\text { durante } 10 \\
\text { semanas; } \\
\text { follow-up de } 8 \\
\text { semanas) } \\
\text { (Hanssen et al., } \\
2017: 2 \text { treinos de } \\
45 \text { minutos/ } \\
\text { /semana, moderada } \\
\text { intensidade; } \\
\text { intervenção durante } \\
12 \text { semanas; } \\
\text { follow-up não } \\
\text { descrito) } \\
\text { C: Ausência de } \\
\text { intervenção }\end{array}$ & $\begin{array}{l}\text { Darabaneanu et al., } \\
2011: \text { - redução da } \\
\text { frequência (dias/ } \\
\text { /mês) [-1,80, IC95\% } \\
\text {-3.43 a -0.17] - } \\
\text { valor com } \\
\text { significância } \\
\text { estatística } \\
\text { - redução da } \\
\text { intensidade da dor } \\
\text { em 20\% e redução } \\
\text { da duração (horas/ } \\
\text { /mês) em 20\% - } \\
\text { valores sem } \\
\text { significância } \\
\text { estatística } \\
\text { Hanssen et al., } \\
2017:- \text { redução da } \\
\text { frequência (dias/ } \\
\text { /mês) em 63\% - } \\
\text { valor sem } \\
\text { significância } \\
\text { estatística }\end{array}$ & $\begin{array}{l}\text { A prática de } \\
\text { exercício aeróbico } \\
\text { parece diminuir o } \\
\text { número de dias } \\
\text { das crises (redução } \\
\text { média de } 0,6 \\
+/-0,3 \text { dias/mês), } \\
\text { a intensidade da } \\
\text { dor e a duração do } \\
\text { episódio }\end{array}$ & 2 \\
\hline $\begin{array}{l}\text { Luedtke et al. } \\
(2016)^{20}\end{array}$ & $\begin{array}{l}\text { Inclusão: adultos } \\
\text { com diagnóstico } \\
\text { de enxaqueca } \\
\text { (critérios de } \\
\text { diagnóstico não } \\
\text { descritos) } \\
\text { Exclusão: outros } \\
\text { tipos de cefaleia }\end{array}$ & $\begin{array}{l}20 \text { EAC: } 2 \\
\text { respondem ao PICO } \\
\text { - Narin et al., } 2003 \\
(n=40) \\
\text { e } \\
\text { - Darabaneanu et al., } \\
2011(n=16)\end{array}$ & $\begin{array}{l}\text { I: Prática de } \\
\text { exercício físico } \\
\text { aeróbico prescrito } \\
\text { por fisioterapeuta } \\
\text { (Narin et al., } 2003 \\
\text { - } 3 \text { treinos/semana } \\
\text { cuja duração de } \\
\text { cada treino não se } \\
\text { encontra descrita; } \\
\text { intervenção e } \\
\text { follow-up de } 8 \\
\text { semanas) } \\
\text { C: Ausência de } \\
\text { intervenção }\end{array}$ & $\begin{array}{l}\text { Narin et al., 2003: } \\
\text { - redução da } \\
\text { frequência (dias/ } \\
\text { /mês) [-3,40; IC95\% } \\
\text {-4,66 a -2,14]; } \\
\text { redução da } \\
\text { intensidade da dor } \\
\text { (escala visual } \\
\text { analógica 0-10) } \\
\text { [-3,00; IC95\% } \\
\text {-3,73 a -2,27]; } \\
\text { redução da } \\
\text { duração (horas/ } \\
\text { /episódio) [-15,80; } \\
\text { IC95\% -23,87 a } \\
\text {-7,73] } \\
\text { Todos os valores } \\
\text { com significância } \\
\text { estatística }\end{array}$ & $\begin{array}{l}\text { A prática de } \\
\text { exercício aeróbico } \\
\text { parece diminuir a } \\
\text { frequência das } \\
\text { crises, a intensidade } \\
\text { da dor e a duração } \\
\text { do episódio. } \\
\text { Não se verificaram } \\
\text { efeitos adversos } \\
\text { da prática de } \\
\text { exercício físico na } \\
\text { prevenção das } \\
\text { crises de } \\
\text { enxaqueca }\end{array}$ & 2 \\
\hline
\end{tabular}




\begin{tabular}{|c|c|c|c|c|c|c|}
\hline \multicolumn{7}{|c|}{ Revisão sistemática } \\
\hline $\begin{array}{l}\text { Referência } \\
\text { bibliográfica }\end{array}$ & $\begin{array}{c}\text { Critérios } \\
\text { inclusão/exclusão }\end{array}$ & Estudos incluídos & $\begin{array}{l}\text { Intervenção (I) e } \\
\text { Comparação (C) }\end{array}$ & Resultados & Conclusão & NE \\
\hline $\begin{array}{l}\text { Gil-Martínez } \\
\text { et al. }(2013)^{7}\end{array}$ & $\begin{array}{l}\text { Inclusão: adultos } \\
\text { com diagnóstico } \\
\text { de enxaqueca } \\
\text { classificados } \\
\text { segundo ICHD-II, } \\
\text { há pelo menos } 6 \\
\text { meses } \\
\text { Exclusão: não } \\
\text { descritos }\end{array}$ & $\begin{array}{l}10 \text { EAC: } 1 \text { responde } \\
\text { ao PICO } \\
\text { - Dittrich et al., } \\
2008(n=30)\end{array}$ & $\begin{array}{l}\text { I: Prática de } \\
\text { exercício físico } \\
\text { aeróbico (Dittrich } \\
\text { et al., } 2008 \text { - } 2 \\
\text { treinos de } 45-60 \\
\text { minutos/semana; } \\
\text { intervenção e } \\
\text { follow-up de } 6 \\
\text { semanas) } \\
\text { C: Ausência de } \\
\text { intervenção }\end{array}$ & $\begin{array}{l}\text { - redução de } \\
\text { frequência (dias/ } \\
\text { /semana/mês/ano) } \\
\text { - valor sem } \\
\text { significância } \\
\text { estatística; } \\
\text { - redução de } \\
\text { intensidade da dor } \\
\text { (escala 0-5) com } \\
\text { p=0,024 - valor } \\
\text { com significância } \\
\text { estatística }\end{array}$ & $\begin{array}{l}\text { Existe evidência } \\
\text { que demonstra } \\
\text { que o exercício } \\
\text { físico aeróbico tem } \\
\text { efeitos benéficos } \\
\text { na enxaqueca, } \\
\text { embora não se } \\
\text { tenha mostrado } \\
\text { superior a outras } \\
\text { intervenções. O } \\
\text { exercício aeróbico } \\
\text { deve ser parte } \\
\text { integrante de uma } \\
\text { abordagem } \\
\text { biopsicossocial de } \\
\text { doentes que } \\
\text { sofrem de } \\
\text { enxaqueca. }\end{array}$ & 2 \\
\hline
\end{tabular}

[Darabaneanu et al., 2011 - 50\%; Hanssen et al., 2017 não descrito] e ainda ausência de dupla ocultação.

Luedtke e colaboradore ${ }^{20}$ concluíram que a prática de exercício aeróbico parece diminuir a frequência das crises, a intensidade da dor e a duração do episódio. Contudo, os estudos incluídos apresentavam amostras pequenas, alguns dos estudos não definiram a duração de cada treino, tendo havido ainda um follow-up inadequado.

\section{Revisões sistemáticas}

Gil-Martínez e colaboradores ${ }^{7}$ concluíram que, apesar de existir evidência limitada de que o exercício físico aeróbico tem efeitos benéficos na enxaqueca, este deve integrar a abordagem biopsicossocial do doente com esta patologia. Este estudo apresenta, porém, uma amostra reduzida, não especifica pormenorizadamente os critérios de seleção dos participantes, quem definiu o programa de exercício nem a percentagem de follow-up, apresentando, assim, fraca qualidade metodológica.

\section{Estudos originais}

Oliveira e colaboradores ${ }^{21}$ demonstraram que o exercício aeróbico reduziu a frequência das crises de enxaqueca no grupo de intervenção, com significância estatística. Este estudo apresentou um curto tempo de seguimento, não tendo sido possível verificar quais os resultados a longo prazo do exercício físico na prevenção da enxaqueca. Apesar de este estudo apresentar uma amostra de conveniência e um número de indivíduos com follow-up completo inferior a $80 \%$, com uma adesão ao programa de exercícios no grupo de intervenção de $67 \%$, revelou qualidade metodológica pelo facto de ser um ensaio aleatorizado, do diagnóstico de enxaqueca ter sido confirmado por um neurologista e da prática de exercício físico ter sido supervisionada por profissionais.

Bond e colaboradores ${ }^{22}$ descreveram que, contrariamente à hipótese inicial do estudo, não se verificou agravamento das características de enxaqueca quando ocorreu redução da prática de exercício físico. Concluíram, assim, não ter sido encontrada associação 


\section{TABELA 4. Estudos originais}

Estudos Originais

\begin{tabular}{|c|c|c|c|c|c|c|c|}
\hline $\begin{array}{l}\text { Referência } \\
\text { bibliográfica }\end{array}$ & $\begin{array}{l}\text { Tipo de } \\
\text { estudo }\end{array}$ & $\begin{array}{l}\text { Critérios } \\
\text { inclusão/ } \\
\text { /exclusão }\end{array}$ & Amostra & Intervenção (I) & Resultados & Conclusão & NE \\
\hline $\begin{array}{l}\text { Oliveira et al. } \\
(2017)^{21}\end{array}$ & ECA & $\begin{array}{l}\text { Inclusão: } \\
\text { mulheres com } \\
\text { enxaqueca } \\
\text { episódica } \\
\text { classificada } \\
\text { segundo ICHD-II, } \\
\text { com idades entre } \\
20 \text { e } 50 \text { anos, } \\
\text { sem terapêutica } \\
\text { profilática, } \\
\text { inativas nos } \\
\text { últimos } 12 \\
\text { meses (prática } \\
\text { de atividade } \\
\text { física em lazer } \\
\leq 1 \text { vez/semana) } \\
\text { Exclusão: rejeição } \\
\text { de participação; } \\
\text { antecedentes de } \\
\text { doença } \\
\text { cardiovascular, } \\
\text { metabólica ou } \\
\text { musculoesque- } \\
\text { lética ou outros } \\
\text { tipos de cefaleia; } \\
\text { qualquer } \\
\text { suplementação } \\
\text { alimentar ou } \\
\text { participação em } \\
\text { outras } \\
\text { intervenções } \\
\text { comportamentais }\end{array}$ & $\begin{array}{l}\text { Mulheres }(n=20) \\
\text { com enxaqueca } \\
\text { episódica } \\
\text { Amostra de } \\
\text { conveniência } \\
\text { Indivíduos } \\
\text { aleatoriamente } \\
\text { alocados nos } 2 \\
\text { ramos } \\
\text { Não se } \\
\text { verificaram } \\
\text { diferenças entre } \\
\text { os grupos } \\
\text { quanto às } \\
\text { características } \\
\text { dos pacientes } \\
\text { incluídos }\end{array}$ & $\begin{array}{l}\text { I: programa de } \\
\text { exercício } \\
\text { aeróbico, } \\
\text { caminhada em } \\
\text { passadeira, } \\
\text { durante } 30 \\
\text { minutos, } 3 \text { vezes } \\
\text { por semana } \\
\text { (follow-up } 12 \\
\text { semanas) } \\
\text { vs } \\
\text { mulheres com } \\
\text { enxaqueca } \\
\text { alocadas ao } \\
\text { ramo } \\
\text { "inatividade", } \\
\text { que não } \\
\text { praticaram } \\
\text { exercício físico } \\
\text { aeróbico }\end{array}$ & $\begin{array}{l}\text { Redução da } \\
\text { frequência } \\
\text { (dias/mês): } \\
\text { - redução no } \\
\text { grupo } \\
\text { intervenção } \\
(-3,8[-5,6 \text { a } \\
9-1,9] ; p=0,001 \text { ) } \\
\text { - valor com } \\
\text { significância } \\
\text { estatística } \\
\text { Adesão ao } \\
\text { programa de } \\
\text { exercícios no } \\
\text { grupo de } \\
\text { intervenção foi } \\
\text { de } 67 \% \\
\text { Não houve } \\
\text { alterações } \\
\text { significativas no } \\
\text { grupo inativo } \\
\text { (média [IC95\% } \\
\text { da diferença] } \\
=0,4[-3,6 \text { a } \\
4,5] ; p=0,8) \text { - } \\
\text { valor com } \\
\text { significância } \\
\text { estatística }\end{array}$ & $\begin{array}{l}\text { O exercício } \\
\text { aeróbico } \\
\text { moderado } \\
\text { promoveu um } \\
\text { efeito preventivo } \\
\text { nas crises de } \\
\text { enxaqueca }\end{array}$ & 2 \\
\hline
\end{tabular}

entre o tipo e duração do exercício físico praticado e as características da crise de enxaqueca. Contudo, descrevem que os resultados não poderão ser generalizados dadas as limitações da amostra, constituída essencialmente por indivíduos do género feminino, raça branca, com obesidade e que pretendiam perder peso. Defendem, assim, que existe a possibilidade da hipó- tese inicial se poder verificar, sendo necessário para isso a realização de novos estudos que avaliem a relação entre o exercício e as características da enxaqueca.

\section{CONCLUSÃO}

A maioria dos estudos selecionados demonstra que existe evidência, ainda que limitada, de efeito 


\begin{tabular}{|c|c|c|c|c|c|c|c|}
\hline \multicolumn{8}{|c|}{ Estudos Originais } \\
\hline $\begin{array}{l}\text { Referência } \\
\text { bibliográfica }\end{array}$ & $\begin{array}{l}\text { Tipo de } \\
\text { estudo }\end{array}$ & $\begin{array}{l}\text { Critérios } \\
\text { inclusão/ } \\
\text { /exclusão }\end{array}$ & Amostra & Intervenção (I) & Resultados & Conclusão & NE \\
\hline $\begin{array}{l}\text { Bond et al. } \\
(2015)^{22}\end{array}$ & $\begin{array}{l}\text { Estudo } \\
\text { caso- } \\
\text {-controlo }\end{array}$ & $\begin{array}{l}\text { Inclusão: } \\
\text { mulheres obesas } \\
\text { com enxaqueca } \\
\text { (segundo } \\
\text { ICHD-III- } \beta \text { ) que } \\
\text { pretendem } \\
\text { participar em } \\
\text { programa de } \\
\text { perda de peso }\end{array}$ & $\begin{array}{l}\text { Mulheres obesas } \\
\text { com enxaqueca } \\
(n=25)\end{array}$ & $\begin{array}{l}\text { Avaliada a } \\
\text { relação da } \\
\text { intensidade da } \\
\text { atividade física } \\
\text { das mulheres } \\
\text { obesas com } \\
\text { enxaqueca com } \\
\text { as diferentes } \\
\text { características da } \\
\text { enxaqueca } \\
\text { (frequência, } \\
\text { intensidade e } \\
\text { duração da crise) } \\
\text { Intensidade } \\
\text { física (leve vs } \\
\text { moderada- } \\
\text {-intensa) foi } \\
\text { monitorizada, } \\
\text { durante } 7 \text { dias, } \\
\text { com banda de } \\
\text { pulso }\end{array}$ & $\begin{array}{l}\text { Não foi } \\
\text { encontrada } \\
\text { relação entre as } \\
\text { variáveis }\end{array}$ & $\begin{array}{l}\text { Não foi } \\
\text { encontrada } \\
\text { relação entre } \\
\text { intensidade do } \\
\text { exercício e as } \\
\text { características } \\
\text { da enxaqueca } \\
\text { avaliadas }\end{array}$ & 2 \\
\hline
\end{tabular}

benéfico do exercício aeróbico no controlo das crises de enxaqueca. Além disso, não foram reportados efeitos adversos significativos que contraindiquem esta prática nos doentes com enxaqueca.

Os estudos incluídos apresentam, contudo, amostras pequenas e heterogéneas, nomeadamente em relação à data e critérios de diagnóstico da enxaqueca, ao género dos indivíduos e às patologias de base. Bond e colaboradores, por exemplo, incidem o seu estudo sobre a população de mulheres obesas, enquanto Oliveira e colaboradores excluem doentes com patologias cardiovasculares e metabólicas, o que dificulta a comparação dos resultados obtidos nos estudos.

Por outro lado, os artigos analisados não são homogéneos quanto aos critérios de diagnóstico da enxaqueca, tipo e duração do exercício físico aeróbico pra- ticado, escalas para avaliação da intensidade de dor e aos períodos de intervenção e de follow-up. A maioria dos estudos refere ainda que seria necessário um período de seguimento mais prolongado para se avaliar os efeitos a longo prazo do exercício físico aeróbico no controlo das crises de enxaqueca.

Pelos motivos expostos, os artigos incluídos apresentam elevada heterogeneidade, pelo que foram classificados com um nível de evidência moderado a baixo. São, assim, necessários mais estudos, nomeadamente com maior uniformização metodológica, para que seja possível definir concretamente a duração adequada da intervenção (número de horas de exercício aeróbico por dia ou por semana), o tipo de intervenção efetuada (diferentes tipos de exercício aeróbico), os benefícios e os efeitos adversos a curto e longo prazo, e os 
subgrupos de doentes com enxaqueca que mais beneficiariam com esta intervenção. Seria ainda importante a definição de uma única escala de avaliação da intensidade da dor a ser utilizada em todos os estudos realizados, reduzindo-se provavelmente assim o risco de viés, conduzindo a que os resultados do efeito da intervenção fossem consequentemente mais fidedignos e comparáveis entre si.

Concluindo, apesar de se considerar necessária a realização de mais ensaios clínicos uniformes e com maior rigor metodológico para definir concretamente os efeitos a curto e longo prazo do exercício aeróbico nos doentes com enxaqueca, é possível recomendar a sua inclusão na abordagem profilática dos mesmos (FR B).

\section{REFERÊNCIAS BIBLIOGRÁFICAS}

1. Espí-López GV, Ruescas-Nicolau MA, Nova-Redondo C, Benítez-Martínez JC, Dugailly PM, Falla D. Effect of soft tissue techniques on headache impact, disability, and quality of life in migraine sufferers: a pilot study. J Altern Complement Med. 2018;24(11):1099-107.

2. Stovner L, Hagen K, Jensen R, Katsarava Z, Lipton R, Scher A, et al. The global burden of headache: a documentation of headache prevalence and disability worldwide. Cephalalgia. 2007;27(3):193-210.

3. World Health Organization. Headache disorders: key facts [homepage]. Geneva: WHO; 2016 Apr 8 [cited 2020 Feb]. Available from: https://www.who.int/news-room/fact-sheets/detail/headache-disorders

4. GBD 2016 Disease and Injury Incidence and Prevalence Collaborators. Global, regional, and national incidence, prevalence, and years lived with disability for 328 diseases and injuries for 195 countries, 1990-2016: a systematic analysis for the Global Burden of Disease Study 2016. Lancet. 2017;390(10100):1211-59.

5. Lipton RB, Bigal ME. Migraine: epidemiology, impact, and risk factors for progression. Headache. 2005;45 Suppl 1:S3-S13.

6. GBD 2016 Headache Collaborators. Global, regional, and national burden of migraine and tension-type headache, 1990-2016: a systematic analysis for the Global Burden of Disease Study 2016. Lancet Neurol. 2018;17(11):954-76.

7. Gil-Martínez A, Kindelan-Calvo P, Agudo-Carmona D, Muñoz-Plata R, López-de-Uralde-Villanueva I, La Touche R. Ejercicio terapéutico como tratamiento de las migrañas y cefaleas tensionales: revisión sistemática de ensayos clínicos aleatorizados [Therapeutic exercise as treatment for migraine and tension-type headaches: a systematic review of randomised clinical trials]. Rev Neurol. 2013;57(10):433-43. Spanish

8. Volcy-Gómez M. Impacto social, económico y en el sistema de salud de la migraña y otras cefaleas primarias [The impact of migraine and other primary headaches on the health system and in social and economic terms]. Rev Neurol. 2006;43(4):228-35. Spanish

9. Pringsheim T, Davenport WJ, Mackie G, Worthington I, Aubé M, Christie SN, et al. Canadian Headache Society guideline for migraine prophylaxis. Can J Neurol Sci. 2012;39(2 Suppl 2):S1-S59.

10. Becker WJ, Findlay T, Moga C, Scott NA, Harstall C, Taenzer P. Guideli- ne for primary care management of headache in adults. Can Fam Physician. 2015;61(8):670-9.

11. Lemmens J, De Pauw J, Van Soom T, Michiels S, Versijpt J, van Breda E, et al. The effect of aerobic exercise on the number of migraine days, duration and pain intensity in migraine: a systematic literature review and meta-analysis. J Headache Pain. 2019;20(1):16.

12. Scher Al, Lipton RB, Stewart WF, Bigal M. Patterns of medication use by chronic and episodic headache sufferers in the general population: results from the frequent headache epidemiology study. Cephalalgia. 2010;30(3):321-8.

13. Probyn K, Bowers H, Mistry D, Caldwell F, Underwood M, Patel S, et al. Non-pharmacological self-management for people living with migraine or tension-type headache: a systematic review including analysis of intervention components. BMJ Open. 2017;7(8):e016670.

14. Koseoglu E, Yetkin MF, Ugur F, Bilgen M. The role of exercise in migraine treatment. J Sports Med Phys Fitness. 2015;55(9):1029-36.

15. Irby MB, Bond DS, Lipton RB, Nicklas B, Houle TT, Penzien DB. Aerobic exercise for reducing migraine burden: mechanisms, markers, and models of change processes. Headache. 2016;56(2):357-69.

16. Geneen LJ, Moore RA, Clarke C, Martin D, Colvin LA, Smith BH. Physical activity and exercise for chronic pain in adults: an overview of Cochrane Reviews. Cochrane Database Syst Rev. 2017;1(1):CD011279.

17. Headache Classification Committee of the International Headache Society. The International Classification of Headache Disorders, 3rd edition. Cephalalgia. 2018;38(1):1-211.

18. Ebell MH, Siwek J, Weiss BD, Woolf SH, Susman J, Ewigman B, et al. Strength of recommendation taxonomy (SORT): a patient-centered approach to grading evidence in the medical literature. Am Fam Physician. 2004;69(3):548-56.

19. La Touche R, Fernández-Pérez JJ, Proy-Acosta A, González-Campodónico L, Martínez-García S, Adraos-Juárez D, et al. Is aerobic exercise helpful in patients with migraine? A systematic review and meta-analysis. Scand J Med Sci Sports. 2020;30(6):965-82.

20. Luedtke K, Allers A, Schulte LH, May A. Efficacy of interventions used by physiotherapists for patients with headache and migraine-systematic review and meta-analysis. Cephalalgia. 2016;36(5):474-92.

21. Oliveira AB, Bachi AL, Ribeiro RT, Mello MT, Vaisberg M, Peres MF. Exercise-induced change in plasma IL-12p70 is linked to migraine prevention and anxiolytic effects in treatment-naïve women: a randomized controlled trial. Neuroimmunomodulation. 2017;24(6):293-9.

22. Bond DS, Thomas JG, O'Leary KC, Lipton RB, Peterlin BL, Roth J, et al. Objectively measured physical activity in obese women with and without migraine. Cephalalgia. 2015;35(10):886-93.

\section{CONFLITO DE INTERESSES}

Os autores declaram não existir quaisquer conflitos de interesse nem qualquer financiamento na realização do presente trabalho de revisão científica.

\section{ENDEREÇO PARA CORRESPONDÊNCIA}

Maria João Coelho

E-mail: maria.joaoccoelho@hotmail.com

https://orcid.org/0000-0003-1341-4897

\section{Recebido em 18-07-2020}

Aceite para publicação em 15-07-2021 


\section{ABSTRACT}

THE ROLE OF EXERCISE IN MIGRAINE TREATMENT: WHAT IS THE EVIDENCE?

Objective: Determine the evidence of the effect of aerobic exercise on the control of migraine attacks.

Data sources: PubMed, National Institute of Health and Care Excellence (NICE), The Cochrane Library, British Medical Journal (BMJ), Canadian Medical Association Infobase, and Centre for Reviews and Dissemination.

Methods: Systematic reviews (SR), meta-analysis (MA), and guidelines were researched in Portuguese, English, and Spanish, using the MeSH terms 'migraine disorders' and 'exercise', with no publication date limit. Original studies (OS) published in the last five years were also researched. Articles that evaluated the effect of aerobic exercise on the frequency, duration, and intensity of migraine attacks were included. The level of evidence (LE) and strength of recommendation (SR) were assigned based on the American Academy of Family Physicians' Strength of Recommendation Taxonomy Scale (SORT).

Results: Sixty-nine articles were obtained with three RS with MA, one SR and two OS met the inclusion criteria. All articles were classified with NE2. Most of the articles support the practice of aerobic exercise as a prophylactic treatment for patients with migraine attacks, however, they have small samples, both short intervention and follow-up periods, and heterogeneous intervention strategies.

Conclusions: Although more clinical studies are needed to assess the long- and short-term impacts of aerobic exercise on migraines, it is possible to conclude that its use could be recommended as prophylactic treatment for these patients (SR B).

Keywords: Migraine disorders; Exercise. 